\title{
Land Evaluation of Old Irrigated Soils in The Middle of Delta Region
}

\author{
Ismail, H. A. ${ }^{1}$ I. Morsy, ${ }^{2}$ B.A. Zamil ${ }^{2}$ and N.I. Talha ${ }^{2}$
}

\begin{abstract}
Quantified land evaluation of soils at Kafer El-Sheikh and El-Gharbia governorates at the middle delta region was carried out. These soils represent 20000 feddan of ElGahwagy area (20000 feddan which located between Kafr El-Sheikh and Qoutur district. Land capability and suitability for different crops were assessed through defining and determining soil physical and chemical properties, irrigation water quality, nutrients status as well as climatic data.
\end{abstract}

ASLE program (Applied System of Land Evaluation) was used to calculate land capability and crop suitability. Results indicate that the soils of the studied area were classified into two land capability classes: class 2 (Good) and class 3 (Faire). Each class has one or more of different sub classes according to the limiting factors.

The limiting factors for land capability are: the relatively low soil permeability, shallow ground water table in some parts, as well as ground water salinity and low levels of soil organic matter and nutrients especially $\mathbf{N}$, $\mathbf{P}$, and $\mathrm{K}$.

Concerning land suitability, different crops can be grown in these soils such as barely, wheat, sugar beet, alfalfa, sunflower, cotton and rice in the order indicated. Other crops can not be cultivated such as pepper, Citrus trees, Date palm, Olive, Fig and Peanut.

\section{INTRODUCTION}

Agricultural production plays an important role in Egypt. It is considered as the source of national income and the way of life for a large part of the population. The agricultural sector in Egypt absorbs $38.2 \%$ of the labor force and able to absorb more. Egyptian Agricultural lands occupy about $4 \%$ (about 8.3 million Feddans) of total area (FAO, 2001). Egypt is now facing a major challenge to increase the rate of growth in agriculture production, to cope a very high annual rate of population increasing $(2.3 \%)$. The national strategy of Egypt aims to adding about 4.32 million Fedden of new land reclamation until year 2017 in different region, based on land suitability and water resources availability (GARPAD, 1997).

According to FAO (1976), land evaluation is the prediction of land performance overtime under specific uses.

Riquier et al (1970) proposed the parametric method of land evaluation and claimed that limitations, as negative and complex concepts in both present and future capability, are better expressed in terms of productivity.

Sys (1979) stated that land evaluation is an opinion, an assessment, a careful judgment, and land evaluation objective is guide wisely the present management and plan the future and best land use among alternatives.

Abd El-Motteleb and Hussein (1985) considered that soil characteristics and environmental conditions are the main factor productivity and land classification. In this system, six soil classes were introduced, based on both soil properties and environmental conditions.

Marie et al (1987) proposed a computer program for land evaluation system (LE) based on that of Abd ElMotteleb and Hussien (1985). This system was modified by EL-Fayoumy (1989) to include soil fertility and irrigation water factor. The last form of this system was developed as a new edition Applied System of Land Evaluation (ASLE) (Morsy, 1994) through adding land suitability to different crops based on land properties as well as climatic data. Each factor was described as an index value to give its statues in the percentage form.

Ismail et al (2001) used ASLE and concluded that Samoul village area (Nile Delta Region) could be classified as good to moderate capability classes. Where Burg El Arab and El-Shahama (western desert) area was Moderate to Marginal capability classes. They also indicated that the main limiting factors were low and high soil permeability, low percentage of clay, shallow ground water table, soil salinity, soil structure, low soil organic matter and nutrients.

Naser Eldin (2001), in his study on Kafr El-Sheikh Governorate soil, found that land capability classes were Excellent, Good, Faire and Poor and the main limiting factors were ground water table, drainage system and nutrients.

Fayed (2003) evaluated the land capability of El Bostan region West Nile Delta. He classified the studied area into two land capability (Moderate and Marginal). $\mathrm{He}$ also stated that, the main limiting soil factor in the studied soils were soil texture, sodium saturation, salinity and carbonate content.

Higab (2005) evaluate some soils of south ElBorolus Lake area. He found that the capability index

\footnotetext{
${ }^{1}$ Soil \& Water Dept., Fuculty of Agric., Alexandria University.

${ }^{2}$ Soils, Water and Env. Res. Inst., Agric. Res. Center, Egypt.

*e-mail: hi_mi99@hotmail.com

Received May5, 2011, Accepted June 30, 2011
} 
for these soils are S2 (Good), S3 (Fair) and N1 (nonagriculture).

Zamil et al, (2009) evaluated 12 thousand feddans at Northern middle Delta at Kafr El Sheikh Governorate. They stated that the area is classified into two land capability cassas; good and fair, the main limiting soil factors were; shallow ground water table in some parts, as well as ground water salinity and low levels of soil organic matter and nutrients. They were suitable for different crops except pepper, olive, fig and peanut

\section{MATERIALS AND METHODS}

\section{Study area:}

The area study is located at the Middle Nile Delta, between Kafr El-Sheikh and Qutour (El-Gharbia Governorate) (Fig.1); the elevation was varied between $2.5 \mathrm{~m}$ and $4.5 \mathrm{~m}$ a.s.1.. This area covers about 20000 feddan, and mainly irrigated by El-Ghwagy canal. It is located at about $3 \mathrm{~km}$ in the south-east by Kafr El-Sheikh city. It has a triangle shape and bounded at the southeast by Samatai drain, South-West by Mit-Yazid canal, and North by Kafr El-Tyfa drain.

This area is irrigated by fresh water from MeetYazid and El-Gahwagy canals and served by tile drainage system.

\section{Field work and laboratory analysis:}

Eighteen soil profiles were selected to represent the study area and georefrenced using GPS. The locations of the studied soil profiles is shown in map (Fig.2).
Soil samples were collected from different soil horizons according to morphological variations or equal distances for homogeneous profiles and were subjected to different physical and chemical analysis: $\mathrm{EC}, \mathrm{PH}, \mathrm{OM}$ and $\mathrm{CaCO} 3$ according to Jackson, 1973; ESP was calculated according to Richard (1954); CEC according to Klute (1986); Available $\mathrm{N}$ and $\mathrm{K}$, according to Cottenie et al (1982); Available P, according to Olsen et al (1954); Mechanical analysis, according to Piper (1950); Hydraulic conductivity (Ks), according to Van Beers (1970); Available water (A.W.) was calculated according to Kulte (1986).

Structure Factor was calculated according to the following formula:

$$
\mathrm{SF}=\left\{1 \frac{\% \text { clay in aggregation analysis }}{\% \text { clay in mechanical analysis }}\right\} \times 100
$$

Also, irrigation water and ground water samples were collected and analyzed for cations and anions; EC and $\mathrm{pH}$. Sodium absorption ratio was calculated as well.

\section{Land evaluation}

Land evaluation and quantified recommendations for soil improvement were implemented using ASLE. This system calculates the land evaluation as a percentage value based on four main factors; soil properties, irrigation water quality, soil fertility and environmental conditions as well as climatic data. The final index of land evaluation (F.I.L.E) was calculated according to Ismail et al (1994).

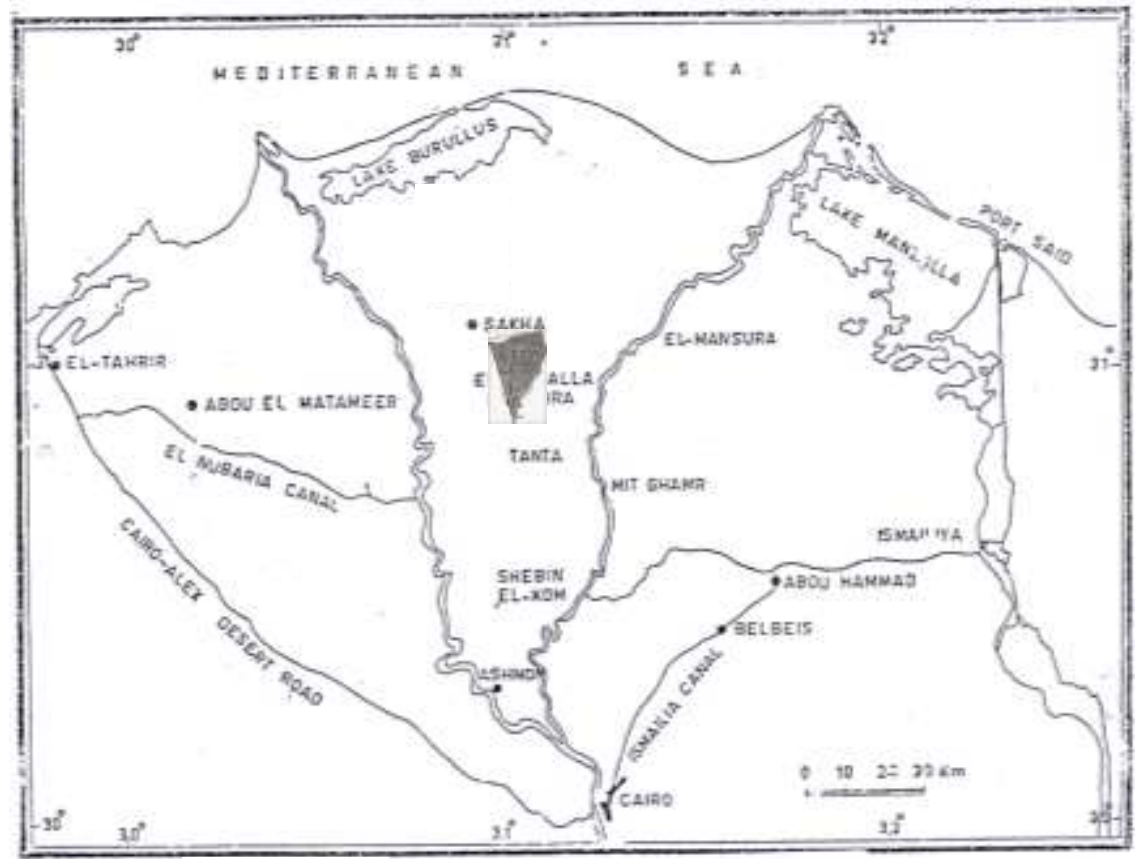

Fig. 1. Location map of studied area 


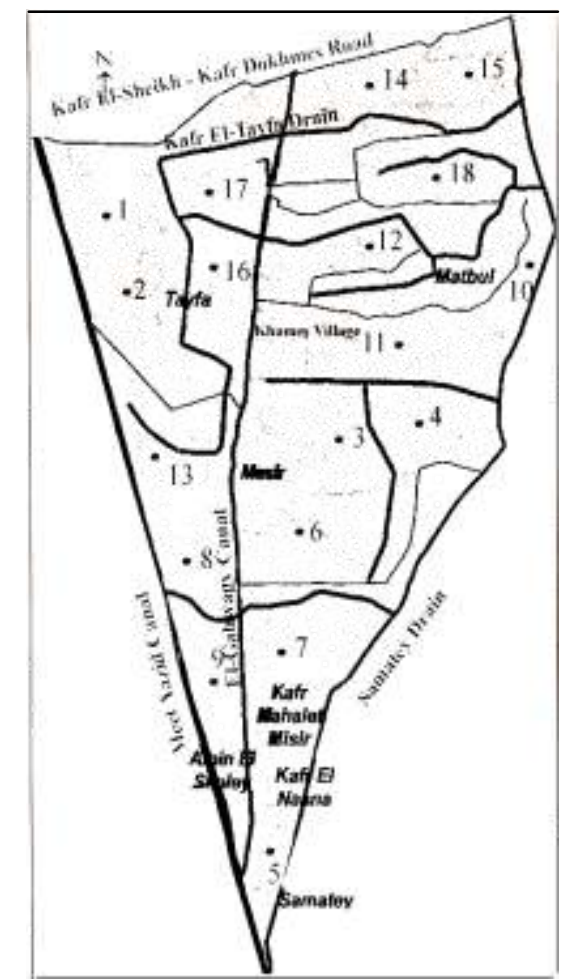

Fig. 2. Location map of the studied profiles.

\section{RESULTS AND DISCUSSION}

\section{Soil characteristics:}

\section{Soil physical properties:}

Data in Table (1) revealed that, clay content ranged between $23.10 \%$ and $58.70 \%$. The soil depth is moderate and ranged from $90 \mathrm{~cm}$ to $120 \mathrm{~cm}$. The soil structure factor ranged from $26.40 \%$ to $43.00 \%$, while the hydraulic conductivity is low and ranged from 0.18 $\mathrm{cm} / \mathrm{h}$ to $4.73 \mathrm{~cm} / \mathrm{h}$. These low values may be attributed to the decrease of organic matter content and higher ESP and SAR values (Madkour et al, 1999). The available water varied from $12.48 \%$ to $23.78 \%$ and it depends on clay and organic matter content.

\section{Soil chemical properties:}

Data in Table (2) showed that, EC values varied from 0.8 to $10.2 \mathrm{dS} / \mathrm{m}$. The $\mathrm{CEC}$ values ranged from 33.81 to 79.80 meq./100g soil. While ESP values ranged from 1.97 to $19.90 \%$; calcium carbonate content varied from $1.20 \%$ to $3.10 \%$ and ground water table salinity varied from 1920 to $5760 \mathrm{ppm}$.

\section{Soil fertility:}

Data in Table (3) revealed that organic matter content is low, where it varied from $0.73 \%$ to $2.36 \%$ the low organic matter content may be due to the increase of decomposition under high temperature in arid and semiarid condition. Concerning the macronutrients, N, P and $\mathrm{K}$ data revealed that they varied from 4.30 to 2.04 ,
105.30 to 41.00 and 12.51 to $251.80 \mathrm{ppm}$ respectively, while exchangeable cations: $\mathrm{K}, \mathrm{Ca}$ and $\mathrm{Mg}$ were varied from 0.11 to $12.16 ; 9.44$ to 1.58 and 56.25 to 37.64 meq/100g soil respectively for the studied soils

\section{Land Capability Classification:}

Data in table (4) indicated that the final index for land evaluation (F.I.L.E) ranges between $52.35 \%$ and $65.87 \%$, so the area could be classified as: $\mathrm{C}_{2}(60-80 \%)$ (good) and $\mathrm{C}_{3}$ (40-60\%) (fair). Concerning the land capability limitation, data revealed that the most limiting factors are soil chemical properties factor and fertility status as; soil organic matter content (OM\%) and available macronutrients (N. P. K).

Accordingly, the main limiting factors for land capability of the studied area at Kafr El-sheikh governorate are ground water depth (GWD) and

hydraulic conductivity $\left(\mathrm{K}_{\mathrm{s}}\right)$ as a physical soil properties, and ground water salinity (GWS) as a chemical soil properties, soil organic matter content (OM\%) and available macro nutrients (NPK) as a soil fertility. However there are no limiting factors concerning either environmental conditions or irrigation water quality.

\section{2- Land suitability for crops:}

The land suitability classes for crops were determined by matching land qualities, climatic data (Table 5) and requirement throughout the suggested computer model. 
Table 1. Physical properties of the studied soils

\begin{tabular}{|c|c|c|c|c|c|c|c|c|}
\hline $\begin{array}{l}\text { P. } \\
\text { no. }\end{array}$ & Depth cm & $\begin{array}{c}\text { Clay } \\
\%\end{array}$ & S.F. & A.W. & $\begin{array}{c}\mathbf{K s} \\
\mathbf{c m} / \mathbf{h r}\end{array}$ & $\begin{array}{l}\text { Prof. depth } \\
\text { cm }\end{array}$ & $\begin{array}{c}\text { G.W.D. } \\
\text { cm }\end{array}$ & $\begin{array}{l}\text { Land } \\
\text { form }\end{array}$ \\
\hline \multirow{3}{*}{1} & $0-20$ & 47.10 & 38.00 & 18.17 & \multirow{3}{*}{.23} & \multirow{3}{*}{90} & \multirow{3}{*}{90} & .2 \\
\hline & $20-55$ & 48.40 & 38.00 & 17.49 & & & & .2 \\
\hline & $55-90$ & 43.50 & 39.00 & 17.30 & & & & .2 \\
\hline \multirow{3}{*}{2} & $0-35$ & 47.20 & 40.00 & 15.84 & \multirow{3}{*}{.31} & \multirow{3}{*}{120} & \multirow{3}{*}{100} & .2 \\
\hline & $35-65$ & 49.30 & 41.00 & 17.07 & & & & .3 \\
\hline & $65-100$ & 46.20 & 40.00 & 17.00 & & & & .2 \\
\hline \multirow{4}{*}{3} & $0-15$ & 48.30 & 43.00 & 17.92 & \multirow{4}{*}{.27} & \multirow{4}{*}{100} & \multirow{4}{*}{90} & .2 \\
\hline & $15-40$ & 44.60 & 39.00 & 18.42 & & & & .2 \\
\hline & $40-70$ & 41.70 & 36.00 & 18.50 & & & & .3 \\
\hline & $70-100$ & 40.80 & 37.00 & 18.60 & & & & .3 \\
\hline \multirow{3}{*}{4} & $0-25$ & 45.80 & 33.00 & 12.48 & \multirow{3}{*}{.40} & \multirow{3}{*}{100} & \multirow{3}{*}{90} & .2 \\
\hline & $25-60$ & 40.70 & 35.00 & 13.70 & & & & .2 \\
\hline & $60-100$ & 36.20 & 35.00 & 14.00 & & & & .2 \\
\hline & $0-20$ & 41.90 & 40.00 & 17.44 & & & & .2 \\
\hline 5 & $20-35$ & 45.00 & 40.00 & 18.94 & .49 & 100 & 90 & .2 \\
\hline & $35-100$ & 43.20 & 38.00 & 19.20 & & & & .1 \\
\hline & $0-20$ & 47.80 & 39.00 & 19.19 & & & & .1 \\
\hline 6 & $20-35$ & 44.30 & 42.00 & 18.38 & .56 & 120 & 100 & .2 \\
\hline & $55-100$ & 40.40 & 42.00 & 18.50 & & & & .2 \\
\hline & $0-15$ & 50.30 & 40.00 & 16.80 & & & & .2 \\
\hline 7 & $15-55$ & 48.20 & 40.00 & 15.77 & 1.71 & 100 & 90 & .2 \\
\hline 7 & $55-100$ & 48.60 & 33.00 & 16.30 & & & & .1 \\
\hline & $0-20$ & 43.30 & 38.00 & 17.12 & & & & .1 \\
\hline 8 & $20-55$ & 44.60 & 40.00 & 14.85 & 2.85 & 100 & 95 & .2 \\
\hline & $55-100$ & 44.20 & 41.00 & 15.30 & & & & .1 \\
\hline & $0-30$ & 48.70 & 42.00 & 14.02 & & & & .2 \\
\hline 9 & $30-70$ & 53.80 & 40.00 & 17.65 & 4.73 & 100 & 95 & .2 \\
\hline & $70-100$ & 26.80 & 32.00 & 18.00 & & & & .2 \\
\hline & $0-35$ & 45.20 & 32.60 & 19.41 & & & & .3 \\
\hline 10 & $35-75$ & 44.30 & 32.60 & 18.90 & .18 & 100 & 90 & .2 \\
\hline & $75-100$ & 44.90 & 31.80 & 18.50 & & & & .1 \\
\hline & $0-35$ & 45.30 & 33.40 & 12.71 & & & & .1 \\
\hline 11 & $35-75$ & 58.70 & 32.80 & 16.67 & 3.85 & 100 & 90 & .2 \\
\hline 11 & $75-100$ & 38.40 & 30.60 & 20.62 & & & & .3 \\
\hline & $0-30$ & 54.10 & 36.20 & 19.90 & & & & .2 \\
\hline 12 & $30-70$ & 55.20 & 37.40 & 18.95 & .41 & 90 & 80 & .2 \\
\hline & $70-90$ & 56.70 & 35.80 & 18.00 & & & & .2 \\
\hline & $0-15$ & 42.60 & 31.40 & 22.19 & & & & .2 \\
\hline 13 & $15-40$ & 45.20 & 30.60 & 21.90 & .36 & 100 & 80 & .2 \\
\hline 13 & $40-100$ & 43.50 & 30.20 & $\begin{array}{l}19.99 \\
\end{array}$ & & & & .2 \\
\hline & $0-30$ & 45.90 & 31.20 & 13.57 & & & & .1 \\
\hline 14 & $30-70$ & 48.20 & 33.10 & 15.52 & 2.55 & 100 & 85 & .2 \\
\hline & $70-100$ & 46.20 & 28.60 & 17.44 & & & & .1 \\
\hline & $0-25$ & 57.40 & 35.20 & 23.51 & & & & .2 \\
\hline & $25-60$ & 55.00 & 35.10 & 22.34 & & 120 & 05 & .1 \\
\hline 15 & $60-95$ & 47.20 & 32.40 & 22.00 & 2.86 & 120 & 95 & .2 \\
\hline & $95-120$ & 23.10 & 26.40 & 22.62 & & & & .2 \\
\hline & $0-15$ & 48.80 & 30.50 & 21.90 & & & & .1 \\
\hline & $15-40$ & 49.70 & 31.20 & 20.31 & & & & .2 \\
\hline 16 & $40-70$ & 46.40 & 31.40 & 21.82 & .20 & 100 & 80 & .2 \\
\hline & $70-100$ & 44.70 & 30.20 & 23.33 & & & & .1 \\
\hline & $0-15$ & 42.10 & 29.60 & 25.80 & & & & .2 \\
\hline 17 & $15-40$ & 43.20 & 29.40 & 23.78 & .38 & 100 & 80 & .2 \\
\hline & $40-100$ & 41.70 & 28.60 & 21.75 & & & & .2 \\
\hline & $0-30$ & 52.40 & 35.20 & 18.25 & & & & .2 \\
\hline 18 & $30-70$ & 44.50 & 32.10 & 16.46 & .45 & 100 & 85 & .2 \\
\hline & $70-100$ & 47.50 & 32.00 & 16.80 & & & & .2 \\
\hline
\end{tabular}

As: GWD: ground water depth ; AW: available water;Ks: Hydrolic coductivity;Sf: Structure cooffition; GWD: Ground water depth. 
Table 2. The chemical properties of the studied soil

\begin{tabular}{|c|c|c|c|c|c|c|c|c|}
\hline P.no. & Depth cm & EC dsm & $\mathbf{P H}$ & $\mathrm{CaCO}_{3} \%$ & Gypsum \% & CEC meq/100g & ESP & GWS ppm \\
\hline \multirow{3}{*}{1} & $0-20$ & 1.02 & 7.80 & 2.30 & 0.1 & 68.36 & 5.10 & \multirow{3}{*}{3840} \\
\hline & $20-55$ & 1.38 & 8.10 & 2.70 & 0.1 & 66.46 & 10.10 & \\
\hline & $55-90$ & 3.05 & 8.10 & 2.40 & 0.1 & 71.90 & 9.40 & \\
\hline \multirow{3}{*}{2} & $0-35$ & 1.15 & 7.30 & 2.40 & 0.1 & 63.01 & 3.20 & \multirow{3}{*}{3200} \\
\hline & $35-65$ & 1.54 & 7.30 & 2.70 & 0.1 & 63.05 & 2.92 & \\
\hline & $65-100$ & 1.51 & 7.30 & 1.80 & 0.2 & 63.00 & 3.02 & \\
\hline \multirow{4}{*}{3} & $0-15$ & 1.02 & 7.90 & 2.10 & 00 & 50.12 & 7.34 & \multirow{4}{*}{1920} \\
\hline & $15-40$ & 1.54 & 7.80 & 2.30 & 0.1 & 55.32 & 6.36 & \\
\hline & $40-70$ & 1.21 & 7.80 & 1.70 & 0.1 & 33.81 & 11.68 & \\
\hline & $70-100$ & .80 & 7.20 & 1.90 & 0.2 & 45.06 & 2.89 & \\
\hline \multirow{3}{*}{4} & $0-25$ & .94 & 8.10 & 2.10 & 0.1 & 43.02 & 16.21 & \multirow{3}{*}{2560} \\
\hline & $25-60$ & 1.37 & 8.10 & 1.70 & 0.1 & 36.44 & 4.12 & \\
\hline & $60-100$ & 1.50 & 8.00 & 1.70 & 0.1 & 38.36 & 5.16 & \\
\hline \multirow{3}{*}{5} & $0-20$ & 3.59 & 8.20 & 1.90 & 0.2 & 46.17 & 10.27 & \multirow{3}{*}{5120} \\
\hline & $20-35$ & 3.68 & 7.70 & 1.70 & 0.2 & 43.18 & 8.20 & \\
\hline & $35-100$ & 10.02 & 8.30 & 1.50 & 0.1 & 43.02 & 19.25 & \\
\hline \multirow{3}{*}{6} & $0-20$ & 2.05 & 8.30 & 2.10 & 00 & 35.46 & 17.90 & \multirow{3}{*}{2560} \\
\hline & $20-55$ & 1.74 & 7.90 & 2.10 & 00 & 43.49 & 13.60 & \\
\hline & $55-100$ & 1.35 & 7.60 & 1.90 & 00 & 46.60 & 10.58 & \\
\hline \multirow{3}{*}{7} & $0-15$ & 4.31 & 7.90 & 2.20 & 0.1 & 44.74 & 15.29 & \multirow{3}{*}{5760} \\
\hline & $15-55$ & 8.40 & 8.10 & 2.70 & 00 & 44.44 & 16.45 & \\
\hline & $55-100$ & 2.99 & 8.20 & 2.90 & 0.2 & 36.34 & 17.62 & \\
\hline \multirow{3}{*}{8} & $0-20$ & 1.98 & 7.40 & 1.70 & 0.2 & 43.82 & 6.27 & \multirow{3}{*}{3200} \\
\hline & $20-60-$ & 2.51 & 7.80 & 1.90 & 0.2 & 40.50 & 12.62 & \\
\hline & $60-100$ & 3.05 & 8.00 & 1.50 & 0.2 & 44.39 & 15.06 & \\
\hline & $0-30$ & 1.54 & 7.30 & 1.40 & 0.2 & 40.81 & 1.97 & \\
\hline 9 & $30-70$ & 1.51 & 7.30 & 1.00 & 0.2 & 39.09 & 4.44 & 2560 \\
\hline & $70-100$ & 1.66 & 7.30 & 1.20 & 0.2 & 40.40 & 3.00 & \\
\hline & $0-35$ & 2.55 & 8.10 & 2.90 & 0.1 & 66.46 & 6.77 & \\
\hline 10 & $35-75$ & 1.95 & 8.30 & 3.00 & 0.1 & 68.37 & 12.72 & 3840 \\
\hline & $75-100$ & 4.48 & 8.30 & 2.90 & 0.1 & 56.48 & 13.63 & \\
\hline & $0-35$ & 1.84 & 8.20 & 1.50 & 0.1 & 58.27 & 15.30 & \\
\hline 11 & $35-75$ & 2.04 & 8.10 & 1.40 & 0.2 & 70.72 & 10.66 & 3200 \\
\hline & $75-100$ & 1.30 & 8.30 & 1.20 & 0.2 & 62.42 & 14.70 & \\
\hline & $0-30$ & 4.15 & 7.80 & 1.50 & 0.1 & 63.48 & 8.50 & \\
\hline 12 & $30-70$ & 3.94 & 8.50 & 1.60 & 0.1 & 79.80 & 18.10 & 3840 \\
\hline & $\begin{array}{l}70-90 \\
\end{array}$ & 3.35 & 8.30 & 1.40 & 0.2 & 70.80 & 15.80 & \\
\hline & $0-15$ & 2.23 & 7.50 & 1.60 & 00 & 71.44 & 5.07 & \\
\hline 13 & $15-40$ & 2.00 & 7.80 & 1.80 & 0.1 & 68.41 & 7.31 & 2880 \\
\hline & $40-100$ & 3.68 & 8.00 & 1.70 & 0.1 & 68.08 & 12.63 & \\
\hline & $\begin{array}{c}0-30 \\
\end{array}$ & 5.85 & 8.20 & 2.60 & 0.1 & 68.80 & 12.60 & \\
\hline 14 & $30-70$ & 3.45 & 8.30 & 2.90 & 0.2 & 60.84 & 17.59 & 4800 \\
\hline & $70-100$ & 2.84 & 8.50 & 3.10 & 0.2 & 46.10 & 19.90 & \\
\hline & $0-25$ & 2.12 & 7.20 & 2.00 & 0.1 & 50.92 & 6.67 & \\
\hline 15 & $25-60$ & 1.80 & 8.00 & 2.00 & 0.1 & 55.20 & 13.80 & 3584 \\
\hline 15 & $60-95$ & 1.79 & 8.00 & 2.10 & 0.2 & 47.80 & 11.90 & 3504 \\
\hline & $90-120$ & 1.59 & 8.10 & 1.90 & 0.2 & 40.60 & 12.72 & \\
\hline & $0-15$ & 2.75 & 7.50 & 2.40 & 0.1 & 61.48 & 5.99 & \\
\hline 16 & $15-40$ & 2.11 & 7.70 & 2.90 & 00 & 61.66 & 8.43 & 2432 \\
\hline 10 & $40-70$ & 2.41 & 8.10 & 3.10 & 0.2 & 52.60 & 14.45 & 2432 \\
\hline & $70-100$ & 2.65 & 8.10 & 2.50 & 0.2 & 62.71 & 12.28 & \\
\hline & $\begin{array}{l}0-15 \\
\end{array}$ & 3.21 & 7.20 & 1.90 & 0.1 & 67.32 & 5.94 & \\
\hline 17 & $15-40$ & 3.56 & 7.30 & 1.50 & 0.1 & 63.58 & 6.98 & 4608 \\
\hline & $40-100$ & 5.99 & 8.10 & 1.80 & 0.1 & 66.43 & 13.55 & \\
\hline & $0-30$ & 6.40 & 7.60 & 2.00 & 0.2 & 58.30 & 15.80 & \\
\hline 18 & $30-70$ & 2.22 & 7.20 & 1.70 & 0.2 & 67.39 & 7.82 & 5120 \\
\hline & $70-100$ & 8.78 & 8.10 & 1.70 & 0.2 & 60.83 & 13.88 & \\
\hline
\end{tabular}


Table 3. The fertility properties of the studied soil

\begin{tabular}{|c|c|c|c|c|c|c|c|c|}
\hline \multirow{2}{*}{ P.no. } & \multirow{2}{*}{ Depth (cm) } & \multirow{2}{*}{$\begin{array}{l}\text { OM } \\
(\%)\end{array}$} & \multicolumn{3}{|c|}{ Available NPK (ppm) } & \multicolumn{3}{|c|}{ Exchangeable (meq/100g soil) } \\
\hline & & & $\mathbf{N}$ & $\mathbf{P}$ & $\mathbf{K}$ & $\mathbf{K}$ & Ca & Mg \\
\hline \multirow{2}{*}{1} & $0-20$ & 2.36 & 9.20 & 4.09 & 105.3 & 0.54 & 44.16 & 20.16 \\
\hline & $20-55$ & 2.04 & 7.80 & 2.10 & 160.7 & 0.49 & 41.25 & 17.51 \\
\hline \multirow{2}{*}{2} & $0-35$ & 2.16 & 12.10 & 7.44 & 140.4 & 0.51 & 47.91 & 12.69 \\
\hline & $35-65$ & 1.98 & 16.20 & 3.66 & 148.3 & 0.61 & 45.83 & 14.77 \\
\hline \multirow{2}{*}{3} & $0-15$ & 2.30 & 8.20 & 6.47 & 206.7 & 1.36 & 35.64 & 9.44 \\
\hline & $15-40$ & 1.76 & 9.10 & 2.50 & 218.4 & 0.84 & 35.64 & 15.32 \\
\hline \multirow{2}{*}{4} & $0-25$ & 2.11 & 4.30 & 5.69 & 120.9 & 0.60 & 22.87 & 20.34 \\
\hline & $25-60$ & 1.88 & 6.50 & 2.70 & 140.8 & 0.65 & 12.16 & 23.33 \\
\hline \multirow{2}{*}{5} & $0-20$ & 2.20 & 28.90 & 7.21 & 140.4 & 0.57 & 45.53 & 14.77 \\
\hline & $20-35$ & 1.90 & 9.60 & 4.37 & 187.2 & 0.52 & 56.25 & 10.41 \\
\hline \multirow[b]{2}{*}{6} & $0-20$ & 2.36 & 28.50 & 6.26 & 218.4 & 1.00 & 34.68 & 18.06 \\
\hline & $20-55$ & 1.96 & 22.60 & 5.90 & 206.5 & 0.87 & 43.76 & 18.86 \\
\hline \multirow{2}{*}{7} & $0-15$ & 2.45 & 20.80 & 10.93 & 175.5 & 1.14 & 24.76 & 28.94 \\
\hline & 1555 & 1.88 & 26.20 & 8.19 & 444.6 & 0.92 & 27.72 & 25.20 \\
\hline \multirow{2}{*}{8} & $0-20$ & 1.97 & 41.00 & 5.78 & 144.3 & 1.24 & 37.51 & 21.07 \\
\hline & $20-60$ & 1.50 & 11.00 & 3.40 & 226.2 & 1.00 & 43.76 & 16.84 \\
\hline \multirow{2}{*}{9} & $0-30$ & 2.05 & 7.00 & 8.09 & 245.6 & 0.81 & 33.66 & 15.34 \\
\hline & $30-70$ & 1.42 & 7.60 & 4.33 & 237.9 & 0.31 & 39.70 & 16.46 \\
\hline \multirow{2}{*}{10} & $0-35$ & 1.86 & 25.20 & 12.51 & 105.3 & 0.56 & 23.76 & 37.64 \\
\hline & $35-75$ & 1.54 & 20.80 & 8.60 & 126.4 & 0.84 & 27.72 & 31.08 \\
\hline \multirow{2}{*}{11} & $0-35$ & 1.95 & 25.00 & 6.64 & 237.9 & 0.88 & 24.70 & 24.20 \\
\hline & $35-75$ & 1.66 & 18.60 & 5.40 & 251.8 & 0.56 & 39.58 & 23.04 \\
\hline \multirow{2}{*}{12} & $0-30$ & 2.11 & 33.60 & 5.58 & 187.2 & 0.84 & 35.64 & 21.60 \\
\hline & $30-70$ & 1.63 & 30.80 & 4.98 & 183.2 & 0.70 & 45.80 & 18.80 \\
\hline \multirow{2}{*}{13} & $0-15$ & 1.86 & 20.40 & 6.35 & 187.2 & 1.16 & 39.58 & 27.08 \\
\hline & $15-40$ & 1.72 & 16.80 & 4.18 & 173.6 & 0.79 & 37.49 & 25.13 \\
\hline \multirow{2}{*}{14} & $0-30$ & .96 & 14.55 & 6.35 & 187.2 & 0.90 & 37.50 & 21.10 \\
\hline & $30-70$ & .73 & 18.60 & 4.18 & 173.6 & 1.14 & 21.78 & 27.22 \\
\hline \multirow{2}{*}{15} & $0-25$ & 1.68 & 18.10 & 4.82 & 173.2 & 0.84 & 31.60 & 15.40 \\
\hline & $25-60$ & 1.42 & 18.20 & 5.50 & 206.4 & 0.50 & 21.80 & 19.40 \\
\hline \multirow{2}{*}{16} & $0-15$ & 2.00 & 12.80 & 8.98 & 175.5 & 0.96 & 28.72 & 28.12 \\
\hline & $15-40$ & 1.72 & 14.60 & 4.36 & 181.4 & 1.58 & 24.76 & 30.12 \\
\hline \multirow{2}{*}{17} & $0-15$ & 1.98 & 14.50 & 5.29 & 140.4 & 0.56 & 35.41 & 23.17 \\
\hline & $15-40$ & 1.66 & 15.60 & 5.14 & 161.5 & 0.87 & 39.58 & 16.98 \\
\hline \multirow{2}{*}{18} & $0-30$ & 2.10 & 19.20 & 2.04 & 120.9 & 0.90 & 24.70 & 24.20 \\
\hline & $30-70$ & 1.90 & 17.80 & 3.51 & 122.6 & 0.11 & 35.64 & 19.24 \\
\hline
\end{tabular}

Table 4. Land capability classes

\begin{tabular}{|c|c|c|c|c|c|c|c|c|}
\hline \multirow{2}{*}{$\begin{array}{l}\text { P. } \\
\text { No. }\end{array}$} & \multicolumn{2}{|c|}{ Soil properties } & \multirow{2}{*}{$\begin{array}{c}\text { Soil } \\
\text { index }\end{array}$} & \multirow{2}{*}{ W.I } & \multirow{2}{*}{ F.I } & \multirow{2}{*}{ E.I } & \multirow{2}{*}{$\begin{array}{l}\text { Final } \\
\text { index }\end{array}$} & \multirow{2}{*}{ Constrains } \\
\hline & P.I & C.I & & & & & & \\
\hline 1 & 62.44 & 76.88 & 48.00 & 96.59 & 33.56 & 69.04 & 53.00 & $G W D, K s, G W S, O M . N P K$. \\
\hline 2 & 67.12 & 79.16 & 53.13 & 96.46 & 39.83 & 66.82 & 57.75 & $K s, G W S, O M, N P K$ \\
\hline 3 & 67.62 & 77.27 & 52.25 & 97.56 & 41.80 & 70.05 & 59.18 & $K s, G W S, O M, N, P$. \\
\hline 4 & 63.16 & 76.55 & 48.35 & 97.90 & 32.26 & 68.87 & 52.35 & $G W D, K s, G W S, O M, N P K$. \\
\hline 5 & 65.77 & 68.77 & 45.23 & 97.61 & 42.42 & 69.04 & 56.81 & $G W D, K s, G W S, E C e, O M, N P K$. \\
\hline 6 & 69.77 & 74.42 & 51.92 & 97.30 & 50.24 & 69.04 & 62.57 & $G W D, G W S, O M, P, K$ \\
\hline 7 & 69.34 & 69.96 & 48.51 & 96.55 & 56.69 & 65.76 & 62.68 & $G W D, G W S, O M, N, P$. \\
\hline 8 & 78.63 & 79.62 & 62.60 & 97.72 & 47.10 & 75.29 & 65.87 & $G W S, O M, P, K$ \\
\hline 9 & 78.99 & 82.97 & 65.54 & 96.16 & 40.57 & 62.17 & 60.25 & $G W S, O M, N, P$. \\
\hline 10 & 63.49 & 75.41 & 47.88 & 97.52 & 45.53 & 68.43 & 59.07 & $K s, G W S, O M, N P K$. \\
\hline 11 & 71.19 & 76.62 & 54.55 & 96.26 & 46.99 & 77.50 & 63.59 & $G W S, O M, N, P$ \\
\hline 12 & 64.45 & 73.10 & 47.11 & 95.32 & 47.54 & 75.03 & 60.54 & $G W D, G W S, K s, O M, P, K$. \\
\hline 13 & 67.37 & 76.23 & 51.36 & 97.61 & 46.59 & 72.33 & 61.53 & $K s, G W S, O M, N P K$ \\
\hline 14 & 69.81 & 71.91 & 50.21 & 97.07 & 40.52 & 70.07 & 57.83 & $G W S, O M, N P K$ \\
\hline 15 & 75.11 & 76.29 & 57.30 & 95.47 & 41.82 & 63.61 & 59.21 & $G W S, O M, N P K$ \\
\hline 16 & 63.88 & 75.88 & 48.48 & 96.76 & 46.08 & 73.20 & 60.31 & $K s, G W S, O M, N P K$. \\
\hline 17 & 68.83 & 73.36 & 50.49 & 97.95 & 43.24 & 63.88 & 58.14 & $K s, G W S, O M, N P K$. \\
\hline 18 & 64.60 & 71.23 & 46.02 & 96.59 & 35.87 & 68.43 & 53.63 & $G W D, K s, G W S, O M, N P K$. \\
\hline
\end{tabular}


Table 5. Climatic data during the period 2000-2008 from Sakha station

\begin{tabular}{|c|c|c|c|c|c|c|c|}
\hline \multirow{2}{*}{ Months } & \multicolumn{3}{|c|}{ Temperature $\mathbf{C}$} & \multirow{2}{*}{ Rain- fall } & \multirow{2}{*}{$\begin{array}{c}\text { Evaporation } \\
\text { mm/month }\end{array}$} & \multirow{2}{*}{$\begin{array}{l}\text { Relative } \\
\text { Humidity }\end{array}$} & \multirow{2}{*}{$\begin{array}{c}\text { Wind Speed } \\
\mathrm{m} / \mathrm{sec}\end{array}$} \\
\hline & Max. & Min. & Mean & & & & \\
\hline January & 18.42 & 6.5 & 12.46 & 13.6 & 61 & 80 & 1.29 \\
\hline February & 19.9 & 7 & 13.45 & 12.8 & 66 & 78 & 1.37 \\
\hline March & 22.7 & 8.6 & 15.65 & 5.91 & 75.01 & 76 & 1.7 \\
\hline April & 26.86 & 10.84 & 18.85 & 2.78 & 90.4 & 69 & 1.41 \\
\hline May & 30.2 & 14.5 & 22.35 & 0 & 107.4 & 65 & 1.2 \\
\hline June & 32.1 & 17.8 & 24.95 & 0 & 119.5 & 64 & 1.1 \\
\hline July & 33.8 & 19.86 & 26.48 & 0.2 & 127.6 & 74 & 1 \\
\hline August & 31.5 & 19.6 & 26.2 & 0.4 & 126 & 76 & 1 \\
\hline September & 28.9 & 18.6 & 25.05 & 0.9 & 119.9 & 75 & 1.2 \\
\hline October & 24.8 & 15.7 & 22.3 & 3.5 & 107.5 & 75 & 1 \\
\hline November & 24.8 & 12.5 & 18.65 & 6.25 & 90 & 77 & 1.02 \\
\hline December & 20.66 & 8.5 & 14.58 & 12.95 & 69.9 & 81 & 1.1 \\
\hline Winter & 19.56 & 9.8 & 16.18 & 9.17 & 64.75 & 77.8 & 1.25 \\
\hline Summer & 31.09 & 16.87 & 23.98 & 0.65 & 115.13 & 70.5 & 1.15 \\
\hline
\end{tabular}

Table 6. Land suitability indices for different field crops

\begin{tabular}{cccccccccc}
\hline P. no. & Wheat \% & Barley \% & $\begin{array}{c}\text { Sunflower } \\
\text { \% }\end{array}$ & $\begin{array}{c}\text { Sugar } \\
\text { beet \% }\end{array}$ & Rice \% & Maize \% & $\begin{array}{c}\text { Faba- } \\
\text { bean \% }\end{array}$ & $\begin{array}{c}\text { Soya } \\
\text { bean \% }\end{array}$ & Cotton \% \\
\hline $\mathbf{1}$ & 93.52 & 93.52 & 34.90 & 92.81 & 90.95 & 35.81 & 33.35 & 31.96 & 35.07 \\
\hline $\mathbf{2}$ & 90.51 & 90.51 & 80.25 & 89.64 & 90.94 & 65.34 & 80.00 & 81.23 & 86.32 \\
\hline $\mathbf{3}$ & 89.91 & 89.91 & 86.39 & 90.15 & 90.34 & 79.99 & 75.64 & 74.50 & 84.08 \\
\hline $\mathbf{4}$ & 89.64 & 89.64 & 86.60 & 90.36 & 90.07 & 80.18 & 74.67 & 75.82 & 84.28 \\
\hline $\mathbf{5}$ & 89.87 & 89.87 & 84.38 & 90.19 & 87.46 & 77.50 & 65.96 & 65.39 & 82.12 \\
\hline $\mathbf{6}$ & 82.37 & 82.37 & 86.12 & 82.27 & 82.77 & 75.86 & 72.95 & 74.06 & 85.00 \\
\hline $\mathbf{7}$ & 80.25 & 82.86 & 88.89 & 81.83 & 33.15 & 69.08 & 66.80 & 68.30 & 85.39 \\
\hline $\mathbf{8}$ & 26.24 & 26.24 & 93.85 & 26.56 & 26.37 & 86.89 & 83.55 & 82.16 & 91.33 \\
\hline $\mathbf{9}$ & 26.67 & 26.67 & 89.57 & 26.17 & 26.80 & 92.48 & 86.13 & 84.70 & 90.00 \\
\hline $\mathbf{1 0}$ & 91.55 & 91.55 & 26.07 & 92.26 & 87.94 & 24.13 & 54.60 & 52.13 & 25.37 \\
\hline $\mathbf{1 1}$ & 26.65 & 26.65 & 92.57 & 26.19 & 26.78 & 85.71 & 82.42 & 81.04 & 90.09 \\
\hline $\mathbf{1 2}$ & 89.43 & 89.43 & 75.15 & 86.34 & 87.03 & 69.58 & 61.95 & 58.23 & 73.14 \\
\hline $\mathbf{1 3}$ & 87.43 & 87.43 & 80.37 & 88.27 & 87.85 & 77.84 & 74.85 & 73.61 & 80.76 \\
\hline $\mathbf{1 4}$ & 26.43 & 26.43 & 93.29 & 26.40 & 25.72 & 82.57 & 73.51 & 72.29 & 90.79 \\
\hline $\mathbf{1 5}$ & 26.84 & 26.84 & 94.83 & 25.99 & 26.97 & 85.04 & 81.77 & 83.02 & 92.29 \\
\hline $\mathbf{1 6}$ & 92.30 & 92.30 & 25.88 & 91.61 & 88.66 & 23.96 & 22.03 & 22.98 & 25.19 \\
\hline $\mathbf{1 7}$ & 87.08 & 87.08 & 80.62 & 88.55 & 84.75 & 78.08 & 71.78 & 70.58 & 81.01 \\
\hline $\mathbf{1 8}$ & 88.39 & 88.39 & 79.61 & 87.43 & 86.02 & 70.46 & 62.73 & 61.69 & 77.47 \\
\hline
\end{tabular}

The data in Table (7) revealed that, those soils are highly suitable for wheat, barley, and sugar beet, sunflower, rice, sorghum, cotton and alfalfa. While it was suitable for fababean, soybean, pear and banana.

From above mentioned discussion. It can be concluded that, the area under consideration is suitable for growing the wheat, barley, sugar beet, sunflower, rice, sorghum, cotton and alfalfa. While it could be used for all crops expect pepper, olive, fig and peanut.

Table (8) indicates that the most of the area was unsuitable for citrus, olive, fig and date palm cultivations.

\section{Spatial distribution of land suitability for some plants:}

Maps (3, 4, 5 and 6) show the land suitability for some selected crops for the study area.

Maps (Fig. 3, 4, 5 and 6) indicate that; most of the area (around $77.97 \%$ ) was highly suitable $(\mathrm{S} 1+\mathrm{S} 2)$ for alfalfa and highly suitable for each one of cotton, sorghum, sunflower and pea representing $88.43 \%$ of the area. Whereas, the high index values occupy $82.29 \%$ and $72.15 \%$ of the area for mais and rice respectively. On the other hand very small area around $2.43 \%$ and $12.09 \%$ was unsuitable for mentioned crops.

For banana and pear; map (Fig. 7) shows that about $61.86 \%$ from the total area is highly suitable while 0.12 $\%$ were unsuitable. 


\section{CONCLUSION}

It could be concluded that the soils of the studied area is classified into two land capability classes: class 2 (Good) and class 3 (Faire). Each class has one or more of different five sub classes according to the limiting factors.

The limiting factors for land capability were the relatively low soil permeability, shallow ground water table in some parts, as well as ground water salinity and low levels of soil organic matter and nutrients especially N.P.K.

Concerning land suitability, different crops can be grown in these soils such as barely, wheat, sugar beet, alfalfa, sunflower, cotton and rice in the order indicated. Other crops can not be cultivated such as pepper, Citrus trees, Date palm, Olive, Fig and Peanut.

Table 7. Land suitability Indices for different Vegetables and Forage Crops

\begin{tabular}{|c|c|c|c|c|c|c|c|c|c|}
\hline P.no. & $\begin{array}{c}\text { Onion } \\
\%\end{array}$ & Cabbage \% & $\begin{array}{c}\text { Pea } \\
\%\end{array}$ & $\begin{array}{c}\text { Potato } \\
\%\end{array}$ & Tomato \% & $\begin{array}{c}\text { Pepper } \\
\%\end{array}$ & $\begin{array}{c}\text { Water } \\
\text { melon \% }\end{array}$ & $\begin{array}{c}\text { Alfalfa } \\
\%\end{array}$ & $\begin{array}{c}\text { Sorghum } \\
\%\end{array}$ \\
\hline 1 & 43.11 & 37.15 & 34.96 & 34.26 & 19.10 & 16.75 & 16.23 & 92.11 & 35.81 \\
\hline 2 & 40.33 & 86.32 & 81.23 & 35.34 & 44.37 & 38.92 & 38.92 & 89.14 & 85.90 \\
\hline 3 & 41.35 & 86.81 & 81.69 & 32.91 & 44.63 & 37.91 & 36.24 & 88.56 & 79.99 \\
\hline 4 & 42.79 & 87.01 & 81.89 & 32.99 & 44.73 & 38.00 & 36.33 & 88.29 & 80.18 \\
\hline 5 & 39.54 & 84.11 & 72.33 & 31.89 & 43.24 & 35.11 & 35.11 & 85.73 & 74.56 \\
\hline 6 & 37.73 & 85.00 & 83.86 & 33.24 & 45.71 & 37.12 & 34.37 & 81.13 & 78.33 \\
\hline 7 & 33.22 & 84.71 & 82.33 & 32.64 & 43.54 & 33.80 & 31.30 & 75.56 & 80.89 \\
\hline 8 & 12.18 & 94.30 & 91.62 & 22.91 & 49.57 & 40.87 & 38.99 & 25.03 & 89.71 \\
\hline 9 & 12.00 & 92.92 & 87.45 & 77.56 & 47.31 & 40.19 & 41.50 & 26.27 & 92.48 \\
\hline 10 & 42.32 & 26.19 & 24.33 & 40.12 & 13.77 & 11.33 & 10.83 & 87.33 & 24.92 \\
\hline 11 & 12.01 & 93.01 & 90.38 & 69.62 & 48.89 & 40.23 & 38.46 & 26.25 & 88.49 \\
\hline 12 & 37.86 & 78.99 & 67.93 & 21.40 & 40.22 & 32.66 & 31.22 & 85.31 & 75.15 \\
\hline 13 & 40.49 & 80.76 & 78.47 & 23.94 & 42.45 & 36.06 & 34.93 & 83.40 & 80.37 \\
\hline 14 & 10.72 & 89.61 & 80.61 & 21.77 & 47.72 & 38.76 & 37.05 & 25.21 & 89.18 \\
\hline 15 & 11.92 & 92.29 & 89.67 & 22.41 & 48.51 & 39.92 & 38.16 & 26.44 & 87.80 \\
\hline 16 & 42.02 & 26.01 & 24.16 & 22.71 & 13.67 & 11.25 & 10.75 & 88.05 & 24.74 \\
\hline 17 & 38.82 & 81.01 & 75.24 & 24.02 & 41.24 & 34.58 & 35.04 & 83.07 & 80.62 \\
\hline 18 & 35.49 & 76.47 & 68.79 & 21.67 & 40.72 & 33.07 & 31.62 & 84.31 & 76.10 \\
\hline
\end{tabular}

Table 8. Land suitability indices for different fruit trees

\begin{tabular}{ccccccc}
\hline P. no. & Citrus $\%$ & Banana & Olive $\%$ & Pear $\%$ & Date Palm $\%$ & Fig $\%$ \\
\hline $\mathbf{1}$ & 32.70 & 80.52 & 14.28 & 79.48 & 31.52 & 30.33 \\
\hline $\mathbf{2}$ & 35.80 & 88.17 & 37.92 & 87.03 & 34.51 & 34.51 \\
\hline $\mathbf{3}$ & 34.87 & 81.18 & 34.95 & 80.05 & 34.70 & 32.13 \\
\hline $\mathbf{4}$ & 34.96 & 80.86 & 35.04 & 79.81 & 34.79 & 32.21 \\
\hline $\mathbf{5}$ & 31.23 & 77.56 & 33.81 & 71.62 & 33.56 & 31.72 \\
\hline $\mathbf{6}$ & 30.08 & 68.79 & 34.23 & 67.90 & 33.55 & 33.60 \\
\hline $\mathbf{7}$ & 27.43 & 59.21 & 32.97 & 58.45 & 31.25 & 27.66 \\
\hline $\mathbf{8}$ & 23.25 & 46.21 & 36.82 & 61.20 & 23.65 & 30.40 \\
\hline $\mathbf{9}$ & 75.23 & 24.64 & 37.96 & 24.32 & 71.81 & 71.81 \\
\hline $\mathbf{1 0}$ & 23.20 & 75.85 & 10.23 & 74.87 & 23.61 & 50.10 \\
\hline $\mathbf{1 1}$ & 70.64 & 22.08 & 36.23 & 21.79 & 71.88 & 66.55 \\
\hline $\mathbf{1 2}$ & 20.10 & 65.58 & 28.56 & 64.73 & 20.73 & 19.81 \\
\hline $\mathbf{1 3}$ & 23.94 & 74.77 & 32.99 & 74.79 & 23.63 & 22.89 \\
\hline $\mathbf{1 4}$ & 20.45 & 19.38 & 36.60 & 19.13 & 22.77 & 21.77 \\
\hline $\mathbf{1 5}$ & 23.80 & 23.26 & 37.70 & 22.96 & 24.22 & 22.42 \\
\hline $\mathbf{1 6}$ & 23.04 & 76.47 & 10.15 & 75.48 & 23.44 & 21.71 \\
\hline $\mathbf{1 7}$ & 22.99 & 72.15 & 33.09 & 71.21 & 23.70 & 22.96 \\
\hline $\mathbf{1 8}$ & 21.99 & 70.00 & 31.23 & 69.09 & 22.67 & 21.67 \\
\hline & & & & &
\end{tabular}




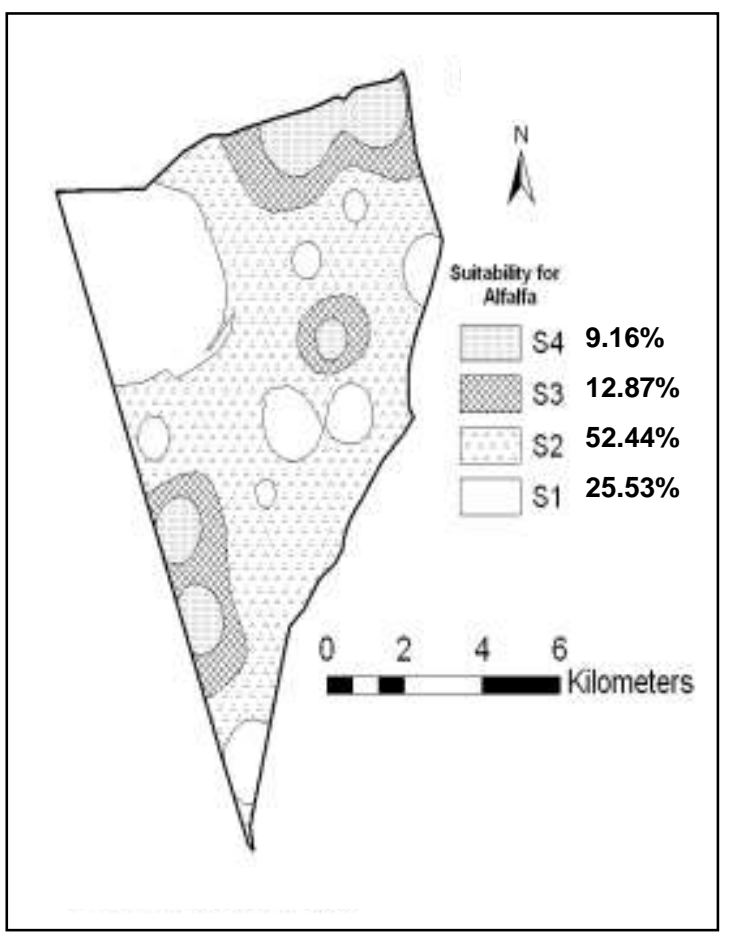

Fig. 3. Map of suitability for Alfalfa

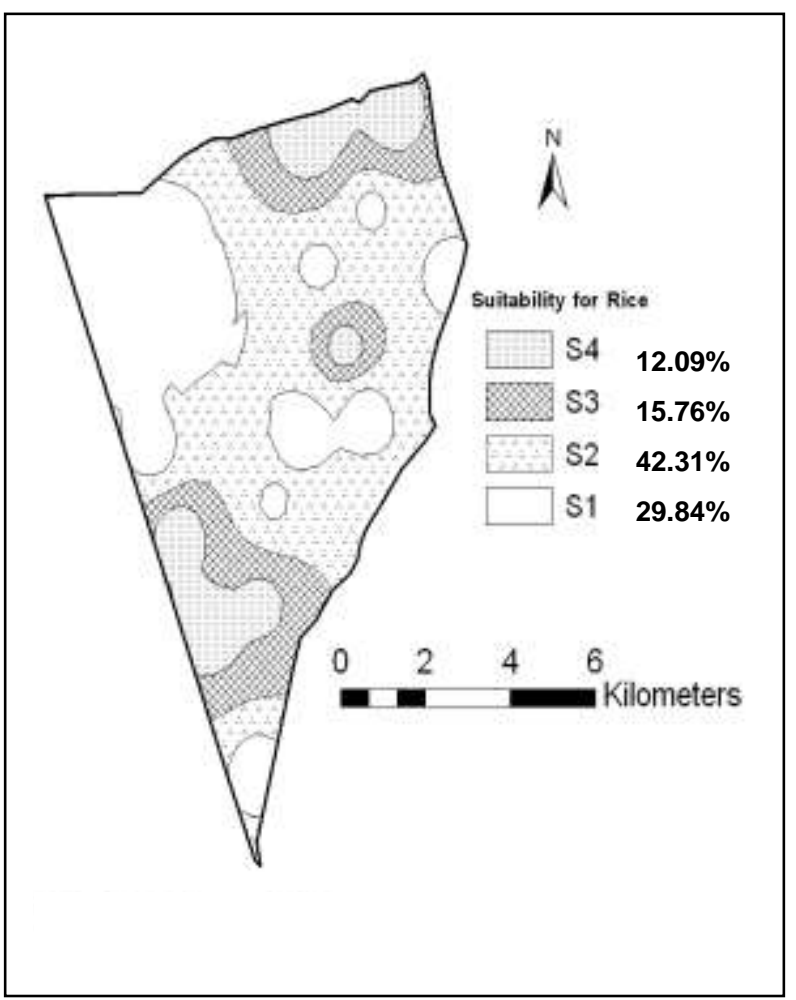

Fig. 5. Suiyability map for Rice

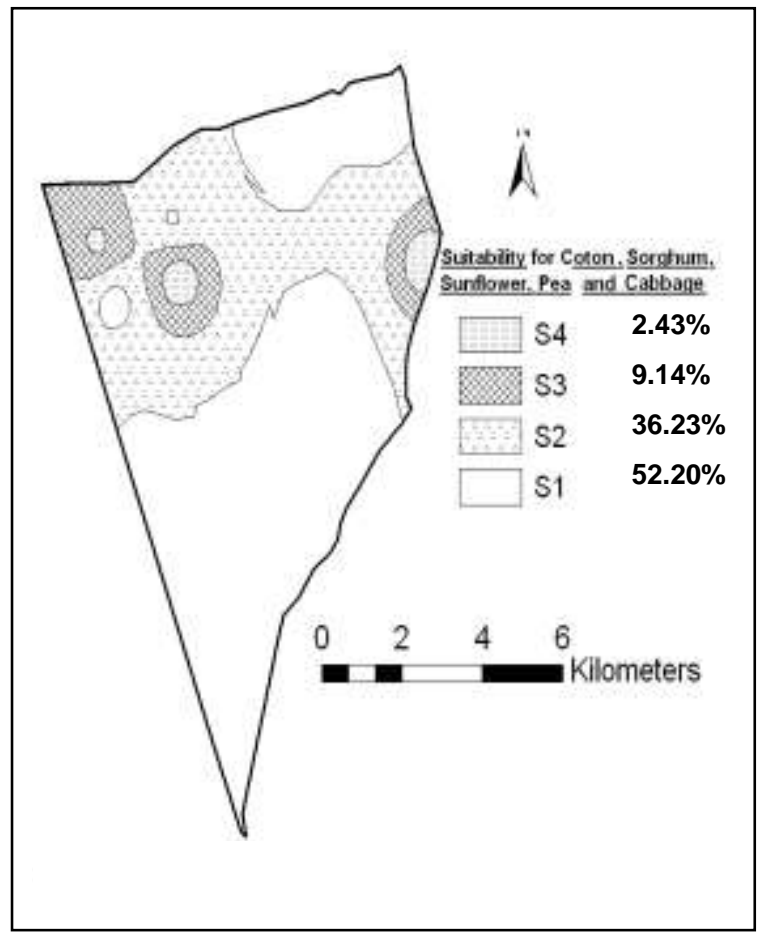

Fig. 4. Map for Cabbage, Pea, Sunflower and Coton

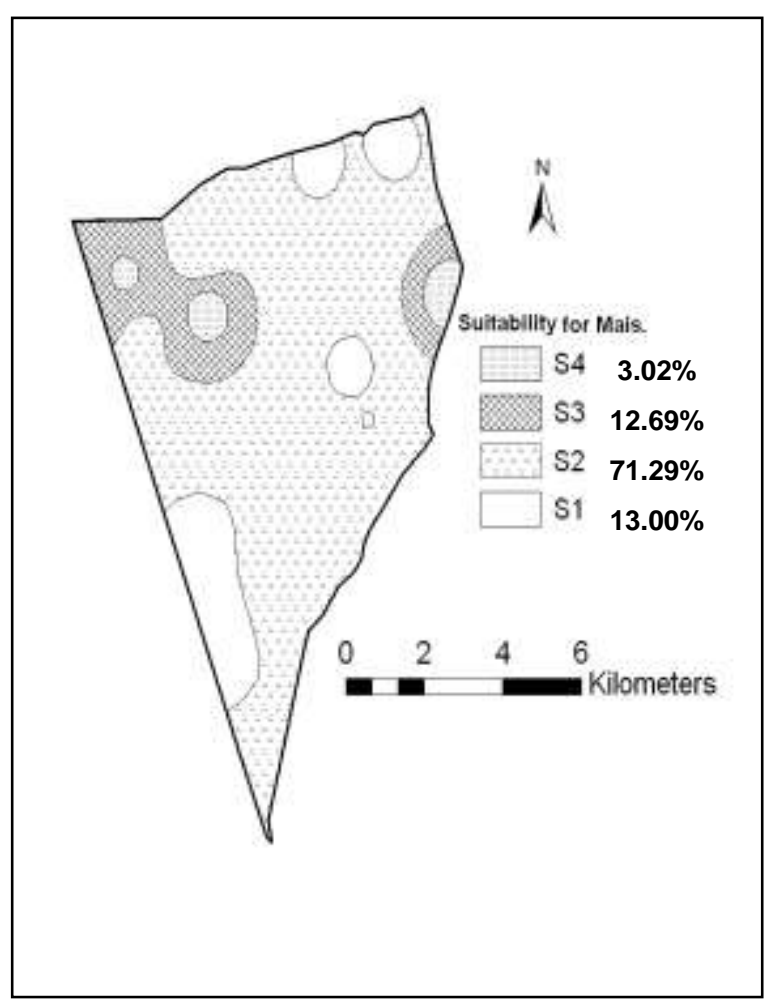

Fig. 6. Suitability map for Mais 


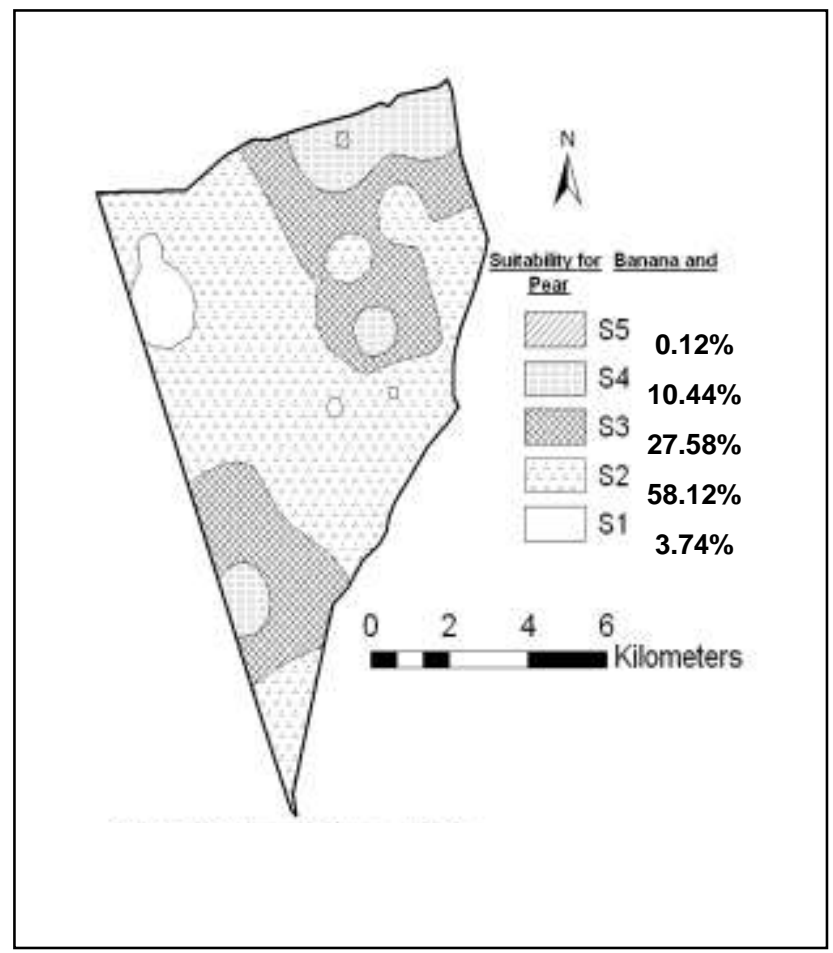

\section{Fig. 7. Suitability map for Banana and Pear}

\section{Recommendation:}

For maximizing the soil productivity of the studied area it is recommended that; increasing the drainage efficiency, through periodical maintenances of title drainage system.

Carrying out sub soiling processes to remove the excess of salts and/ or hard pans which may exist in such heavy clay soil.

Deep plowing shout be carried out to prevent the upward movement of saline ground water to the soil surface through capillary rise.

Application of organic matter and soil amendments to improve physical soil properties and nutrient statues.

Proper fertilization (type, time, amount and place of application) must be followed under the saline soil condition.

\section{RE FERENCES}

Abdel Mottaleb M. A. and S. A.Hussein (1985). Evaluation of Agricultural Land (in Arabic), Faculty of Agriculture, El Azhar University.

Cottenie, A.M. Nerloo, G. Velghe and L. Kiekene (1982). Biological analytical aspects of soil pollution. Lab. of analytical Agro. State Univ. Ghent- Belgium

EL-Fayoumy, M.F. (1989). New approach of land evaluation of some Egyptian regions. Ph.D. Thesis, fac. of Agric. univ. of Alexandria.

FAO (1976). A framework for land evaluation. Solis Bull. No32, FAO, Rome.
FAO (2001). Agriculture trade and Rual Department in the Middle East and North Africa. Recent Department and prospects. The World Bank international economics department.

Fayed, R.I.M. (2003). Impact of land management practices on soil, El-Bostan Region, Egypt. ph. D. thesis- Fac. Agric Alex. univ.

GARPAD, 1997). General Authority for Reclamation projects and development. Strategy for horizontal land reclamation in Egypt until year 2017, Ministry of Agriculture, Egypt. (In Arabic).

Higab, I.A. (2005). An evaluation of some soils of south ElBorolous lake Area. PhD thesis, Fac. Agric Al-Azhar univ.

Ismail, H.A., S.M. Marei and M.E. El-Fayoumy (1994) A modify approach for land evaluation under arid condition. I. Basis and Computer program. J. Agric. Sci., Mansoura Univ., 19 (10): 3483 - 3495.

Ismail H.A., I.M..Morsy, E.M. EL-Zahaby and F.S. El-Nagar (2001). A Developed Expert System for land use planning by coupling Land Information System (LIS) and Modeling. Alex. Agric. Res. 46(3):141-154.

Jackson, M.L. (1973). Soil chemical analysis. Advanced course Ed.2. A Manual of methods useful for instruction and research in soil chemistry, physical chemistry of soil, soil fertility and soil genesis. Revised from Original Edition (1955)

Klute, A. (ed.) (1986). Methods of soil analysis (part1). Physical and mineralogical methods ( ${ }^{\text {nd }}$ Ed.). American society of agronomy. Inc. Soil Sci. of Agron. Inc. Madison. Wisconsin, USA. 
Madkour, H.E.A., A.M.M. Hamouda and S.A. Moussa (1999). Hydrulic conductivity and structure factor of alluvial soils as affected by some soil properties. J. Agric. Sci Mansoura Univ, 24(8): 4249-4258

Marei, S.M., H.A. Ismail and H.Meshref (1987). A. computer program for land evaluation in Egypt. J. Agric. Sci. Mansoura Univ. 12 (4):1427-1447.

Morsy, I.M (1994). A model relating soil properties and variability to land capability and suitability for crops in arid irrigated region .PhD thesis. fac. of Agric ,Univ. of Alexandria.

Nasr EL-Din, I.E.I. (2001). Evaluation of some different soils from Kafer EL-Sheikh under different crop patterns .Ph.D.Thesis,fac.of Agric. Kafer El-Sheikh, Tanta Univ.

Olsen, S, R; C. V. Cole; F. S. Watambe and L. A. Dean ( 1954 ). Estimated of available phosphorus in soil by sodium bicarbonate. U. S. Dept. Agric. Cire. 939.

Page, A.L. (1982). Methods of soil analysis part2: Chemical and microbiological properties (2nd ed.) Amer. Soc. Agron. In soil Sci. soc. Amer. Inc. Madison, Wisconsin, UAA Chapter12:pp. 199-223.
Piper, C.S. (1950). Soil and Plant Analysis. Inter. Sci. Public. Inc. New York.

Richards, R.L. (ed.) (1954). Diagnosis and improvement of saline and alkali soils. Agriculture Hand Book No. 60, U.S Govt. Printing Office, Washington, USA.

Riquier, j., D.L .Bramao and J.P. Cornet (1970). A new system of soil appraisal in terms of actual and potential productivity .FAO soil resource, development and conservation service, land and water development Division, FAO, Rome.

Sys, C. (1979). Evaluation of the physical environment for irrigation in terms of land characteristics and land.

Van Beers, W.F.J. (1970). The auger hole method. Bull. No 8, 11. RI Wageningen, the Netherlands.

Zamil, B.A; M.A.A. Abbd Allah, G.M. Abd El- Salam, M.I. El-Shahawy (2009). Land evaluation of old irrigated soils in north delta region (Rewena area) at Kafr El Sheikh governorate. Alexandria Science Exchange Journal. Vol. 30 No. 2: 214-228. 


\section{الملخص العربي}

\section{تقييم الأراضي المروية القديمة في منطقة وسط الدلتا}

حسن احمد اسماعيل، ايهاب محرم مرسى، بهجت عبد القوى زامل, ناصر ابراهيم طلحة

$$
\begin{aligned}
& \text { تم تقييم الأراضي القديكة الجماورة لترعة القهوجي والتى تقع ما بين ومن الممكن ترتيب ملائمة التربة للمحاصيل في الترتيب التنازلي }
\end{aligned}
$$

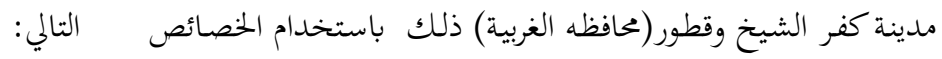

$$
\begin{aligned}
& \text { الشعير > القمح > بنجر السكر > عباد الشمس > القطن > } \\
& \text { الكيماوية والطبيعية وموقف المغذيات وكذلك خصائص مياه الري الرئي } \\
& \text { الأرز. } \\
& \text { ولتعظيم الأنتاجيه في هذه الأراضي موضع الدراسة يجب إتباع }
\end{aligned}
$$

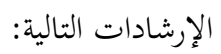

$$
\begin{aligned}
& \text { 1- زيادة كفاءة الصرف بالصيانة الدورية لنظام الصرف المغطى. }
\end{aligned}
$$

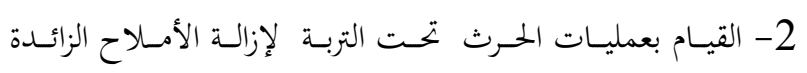

$$
\begin{aligned}
& \text { والطبقات الصماء وبخاصة في الأراضي الطينية الثقيلة. }
\end{aligned}
$$

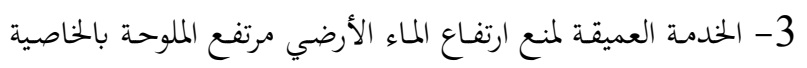

$$
\text { الشعرية إلى سطح التربة. }
$$

4- أضسافه المـادة العضوية وحسـنات التربـة لتحسين خـواص التربة

$$
\text { الفزيائيه وحاله المغذيات. }
$$

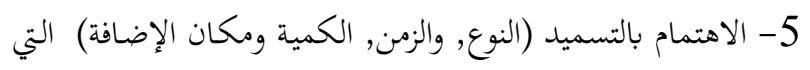

$$
\text { يجب إن تضاف في حاله الأراضى الملحية. }
$$

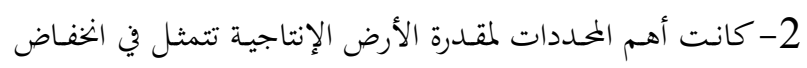

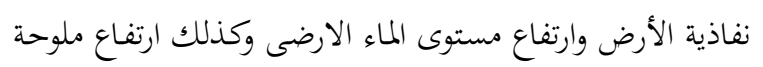

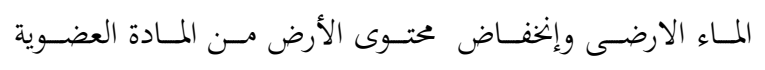
والمغذيات, خاصة النتروجين والفسفور والبوتاسيوم.

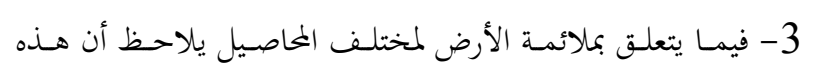

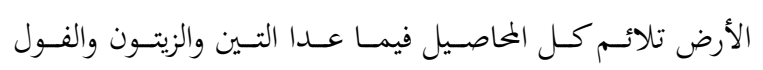

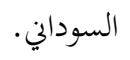

